\title{
Development and Validation of an Adsorptive Method for Aluminium Determination in Fluids Used in Renal Dialysis
}

\author{
Miriam B. Quirós ", Biyun Z. Wu \\ Centro de Electroquímica y Energía Química y Escuela de Química, Universidad de \\ Costa Rica, Ciudad Universitaria Rodrigo Facio, Apartado 2060, Costa Rica
}

Received 31 May 2004; accepted 9 January 2005

\begin{abstract}
It was developed and validated an analytical methodology able to quantify aluminium in haemodialysis fluids, based on the reaction of aluminium with cupferrron (ammonium salt of N-nitroso phenyl hydroxyl amina), on the adsorption of the complex on a hanging drop mercury electrode, and on the measurement of the reduction current of the complex. Parameter validation was done using square wave voltammetry for accumulation potential, time of accumulation, frequency, pulse amplitude, equilibrium time, cupferron concentration, optimum $\mathrm{pH}$. Performance parameters of the method were established: detection limit, quantification limit, reliability, lineal interval for the calibration curves. The method was applied to haemodialysis mineralized fluids, being done the quantification of the aluminium presented.
\end{abstract}

Keywords: fluids, renal dialysis, quantitative analysis, aluminium, adsorptive voltammetry, square wave, cupferron.

\section{Introducción}

El aluminio por muchos años ha sido señalado como causante de daños y enfermedades óseas y neurológicas en encefalopatías. Existen diversas fuentes de contaminación con aluminio, como el aluminio del agua utilizada para preparar fluidos de diálisis y enlazantes de fosfatos. Se ha asociado la contaminación

\footnotetext{
* Corresponding author. E-mail address: mbarquero@ calzada.equi.ucr.ac.cr
} 
con aluminio con ciertos tipos de anemia de diálisis; debido a eso la "Legislación Internacional sobre Diálisis" ha elaborado una serie de regulaciones sobre la calidad del agua, ya que un paciente de hemodiálisis está expuesto a volúmenes de fluidos de diálisis de 20000-30000 litros/año y existe el riesgo de complicaciones agudas o crónicas. La acumulación en hemodiálisis resulta de la contaminación de los fluidos empleados con aluminio y se ha relacionado la acumulación ósea de este metal con la osteodistrofia de diálisis; también se ha demostrado relación entre la acumulación de aluminio y la de peróxidos lipídicos en células rojas de pacientes de hemodiálisis [1]. Se han propuesto diversos tratamientos para la purificación química y microbiológica del agua utilizada en los fluidos de diálisis [2].

Se ha dado importancia a la contaminación microbiológica de las aguas empleadas, pero no se han evaluado completamente todos los parámetros fisicoquímicos de las aguas empleadas para preparar las disoluciones utilizadas durante la hemodiálisis [3].

Se ha reportado una concentración de aluminio en suero de pacientes de hemodiálisis más alta que la concentración del metal en agua, siendo este efecto más pronunciado cuando han sufrido hepatitis [4]. El valor máximo permitido para este metal por la AAMI (American Association for the Advancement of Medical Instrumentation) es de $10 \mu \mathrm{g} / \mathrm{L}$, por lo que el desarrollo de un método de adecuada sensibilidad para su cuantificación es importante [5]. El método propuesto para realizar la cuantificación consta de la reacción de $\mathrm{Al}$ con cupferrón, adsorción sobre el electrodo de $\mathrm{Hg}$, desorción y medida de la corriente producida por la reducción del complejo [6]. Para aplicarlo a muestras reales se debe realizar una previa mineralización de la muestra y regular el pH a 7.0.

También se ha reportado el análisis de disoluciones empleadas en hemodiálisis por medio de preconcentración con resinas de intercambio catiónico y espectrofotometría [7].

Se han estudiado y comparado procedimientos alternos de mineralización en fluidos de hemodiálisis por medio de la determinación polarográfica con diferencial de pulso (DPP) y la absorción atómica con horno de grafito [8]. 


\section{Parte experimental}

Se utilizó un polarógrafo PARC modelo 384 B acoplado a un sistema de tres electrodos $303 \mathrm{~A}$, constituido por un electrodo de gota suspendido, un electrodo de plata/cloruro de plata como referencia y un electrodo de platino como indicador. El software empleado fue desarrollado específicamente para controlar el equipo utilizado [9].

La cristalería empleada se colocó por una semana en $\mathrm{HNO}_{3} 20 \% \mathrm{v} / \mathrm{v}$, luego se reflujó con $\mathrm{HNO}_{3} / \mathrm{HCl}$ 1:3, se enjuagó con abundante agua de un sistema Milli Q, conectado a un destilador y un desionizador. Se utilizó un buffer constituido por con HOAC 2 mol/L ajustado a pH 7.0 con $\mathrm{NaOH}$, ambos reactivos de calidad Suprapur, del que se adicionó $1.00 \mathrm{~mL}$ en la celda polarografica. El cupferrón $0.060 \mathrm{~mol} / \mathrm{L}$ de calidad para análisis se preparó diariamente, empleando agua Milli Q desoxigenada por 10 minutos con nitrógeno y se adicionó $\mathrm{NH}_{3}$ de calidad Suprapur al 2\%; con este procedimiento se logró estabilizar la forma y el potencial del pico. Se adicionaron $40 \mu \mathrm{L}$ de la disolución de cupferrón en la celda, se desoxigenó y se midió por SWV.

\section{Resultados y discusión}

El proyecto comprendió tres etapas: optimización de las condiciones experimentales de medición, validación del método desarrollado y determinación del proceso de digestión adecuado para los fluidos de hemodiálisis. La validación de los parámetros para voltamperometría de onda cuadrada (SWV) se realizó para tiempo de equilibrio, concentración de cupferrón, frecuencia, tiempo de acumulación, potencial de acumulación, amplitud de pulso. En la Fig. 1 se muestra la variación de la corriente con la concentración de cupferrón. Se seleccionó la concentración de $2 \times 10^{-4} \mathrm{~mol} / \mathrm{L}$ para disminuir el valor de los blancos; en la Fig. 2 se muestra la variación de la corriente con la frecuencia, se seleccionó $25 \mathrm{~Hz}$ para disminuir la corriente residual. El tiempo de acumulación escogido fue 60 segundos, de acuerdo a la Fig. 3. La selección del potencial de adsorción del complejo al electrodo se realizó con la Fig. 4: se observa que 
después de -0,4 Voltios la corriente disminuye su valor. En la Fig. 5 se muestra la variación de corriente con la amplitud de pulso, el valor seleccionado fue de 20 $\mathrm{mV}$.

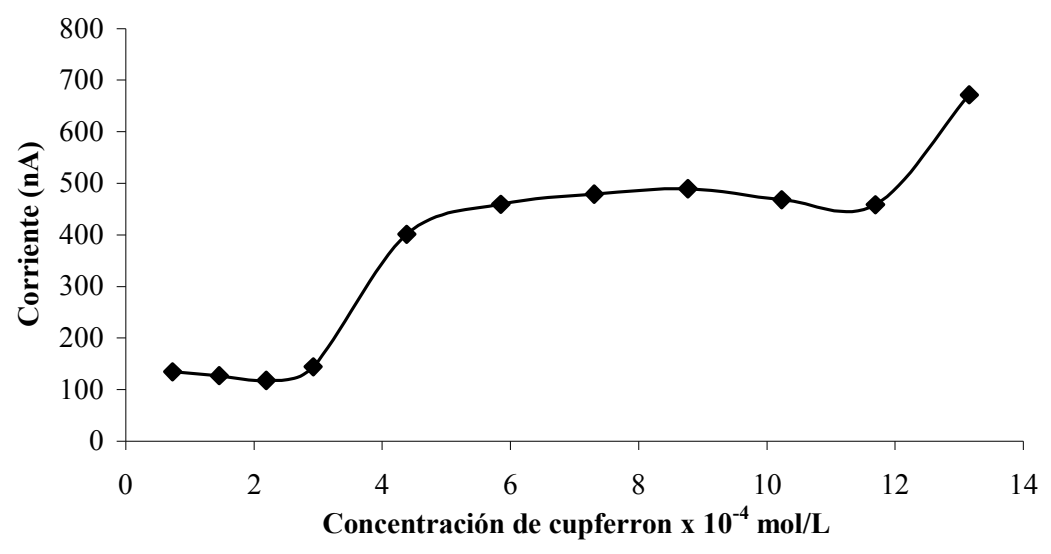

Figura 1. Variación de la corriente con la concentración de cupferrón para $20 \mu \mathrm{g} / \mathrm{L}$ de aluminio adicionados en celda.

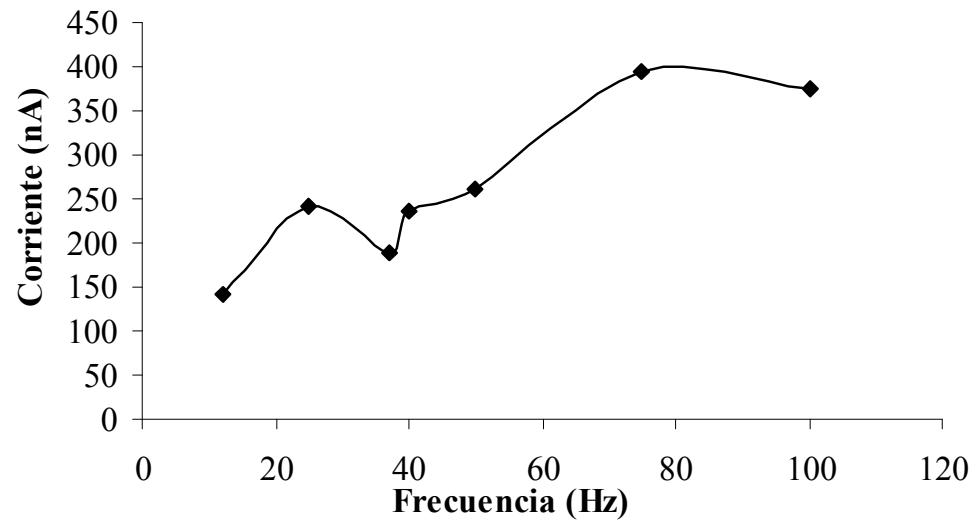

Figura 2. Variación de la corriente con la frecuencia para $20 \mu \mathrm{g} / \mathrm{L}$ de aluminio adicionados en celda. 
Previamente se seleccionó entre diferencial de pulso y voltamperometría de onda cuadrada ya que los valores de corriente para una misma concentración en onda cuadrada eran mayores.

Los valores de los parámetros experimentales optimizados se muestran a en la Tabla 1.

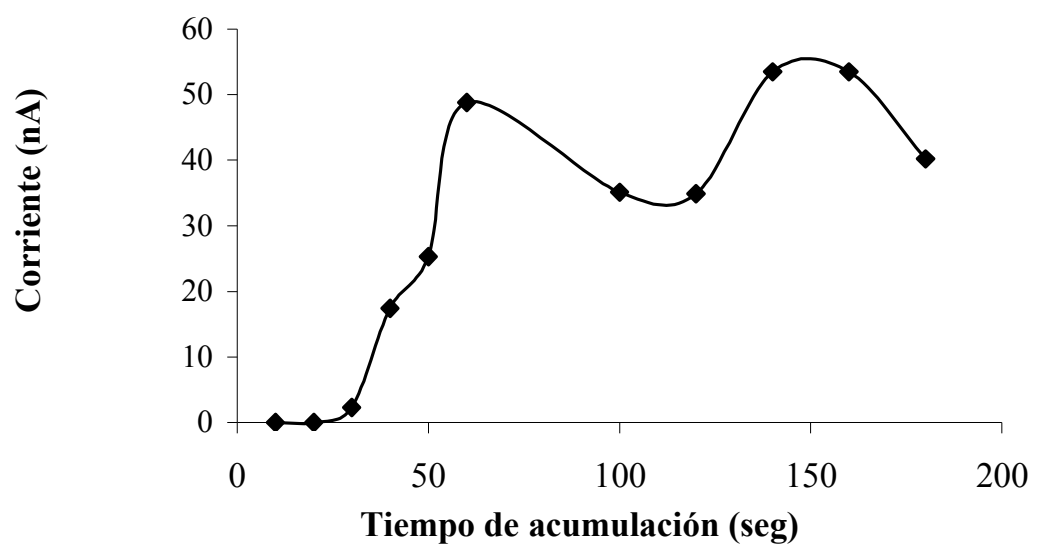

Figura 3. Variación de la corriente con el tiempo de acumulación para $2 \mu \mathrm{g} / \mathrm{L}$ de aluminio adicionados en celda.

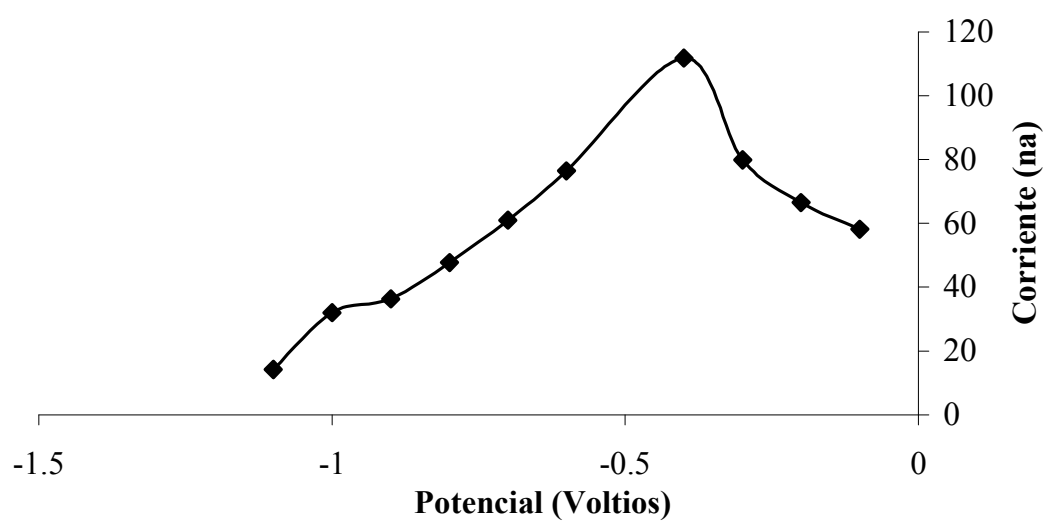

Figura 4. Variación de la corriente con el potencial de acumulación para $2 \mu \mathrm{g} / \mathrm{L}$ de aluminio adicionados en celda. 
La determinación del $\mathrm{pH}$ adecuado se realizó antes de optimizar los parámetros experimentales. Se prepararon una serie de disoluciones reguladoras de diferente pH y se observó donde la forma de la señal era un pico adecuado para cuantificar; algunos voltamperogramas con diferentes $\mathrm{pH}$ se muestran a continuación. Se observó un desplazamiento del potencial del pico hacia potenciales positivos al disminuir el valor de $\mathrm{pH}$.

Tabla 1.Valores optimizados para la determinación de aluminio.

\begin{tabular}{lc}
\hline Potencial de acumulación & $-0.4 \mathrm{~V}$ \\
Tiempo de acumulación & $60 \mathrm{~s}$ \\
Amplitud de pulso & $20 \mathrm{mV}$ \\
Frecuencia & $25 \mathrm{~Hz}$ \\
Tiempo de equilibrio & $20 \mathrm{~s}$ \\
Concentración de cupferrón & $2 \times 10^{-4} \mathrm{~mol} / \mathrm{L}$ \\
pH & 7.00 \\
\hline
\end{tabular}

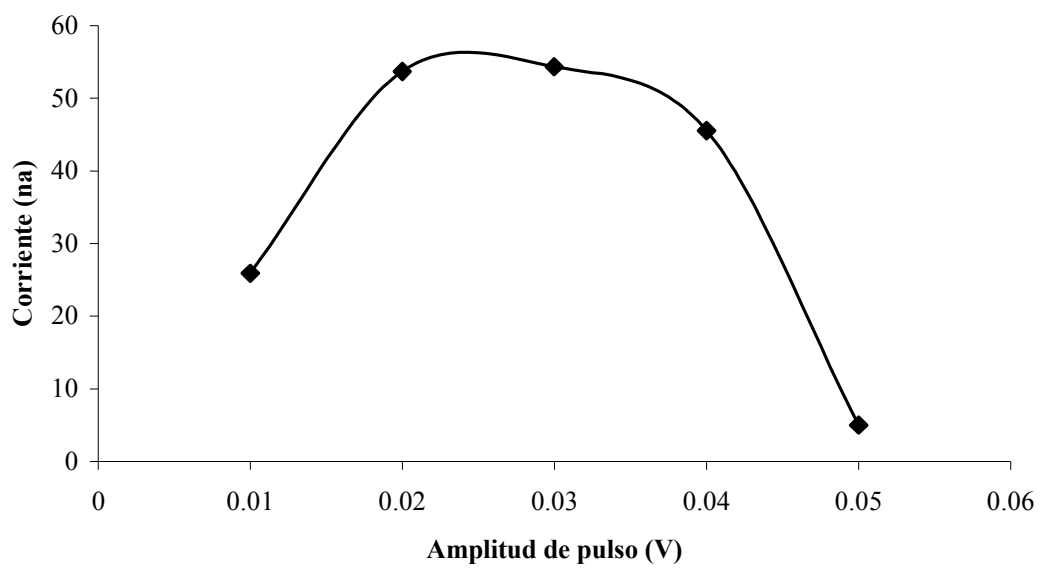

Figura 5. Variación de la corriente con la amplitud de pulso para $2 \mu \mathrm{g} / \mathrm{L}$ de aluminio adicionados en celda.

En la Tabla 2 se muestran las condiciones optimizadas instrumentales de medición. 
Se seleccionó el $\mathrm{pH}$ de 7.0 porque la señal era definida al potencial de -1.3 Voltios, como se muestra en la Fig. 6.

Se determinó el incremento de corriente al adicionar aluminio al potencial de formación del complejo, como se observa en la Fig. 7.

Tabla 2. Condiciones instrumentales en la determinación de aluminio con cupferrón. Determinación del pH adecuado de formación del complejo aluminio cupferrón.

\begin{tabular}{lc}
\hline Técnica & $\mathrm{SWV}$ \\
\hline Potencial inicial & $-0.4 \mathrm{~V}(\mathrm{Ag} / \mathrm{AgCl})$ \\
Potencial final & $-1.4 \mathrm{~V}(\mathrm{Ag} / \mathrm{AgCl})$ \\
Potencial de acondicionamiento & $0 \mathrm{~V}(\mathrm{Ag} / \mathrm{AgCl})$ \\
Amplitud de pulso & $0.020 \mathrm{~V}$ \\
Tiempo de desoxigenación inicial & $200 \mathrm{~s}$ \\
Tiempo de desoxigenación entre réplicas & $20 \mathrm{~s}$ \\
Tiempo de preconcentración & $60 \mathrm{~s}$ \\
Tiempo de acondicionamiento & $0 \mathrm{~s}$ \\
Tiempo de equilibrio & $20 \mathrm{~s}$ \\
Incremento de barrido & $2 \mathrm{mV}$ \\
Frecuencia & $25 \mathrm{~Hz}$ \\
Ciclos & 1 \\
\hline
\end{tabular}

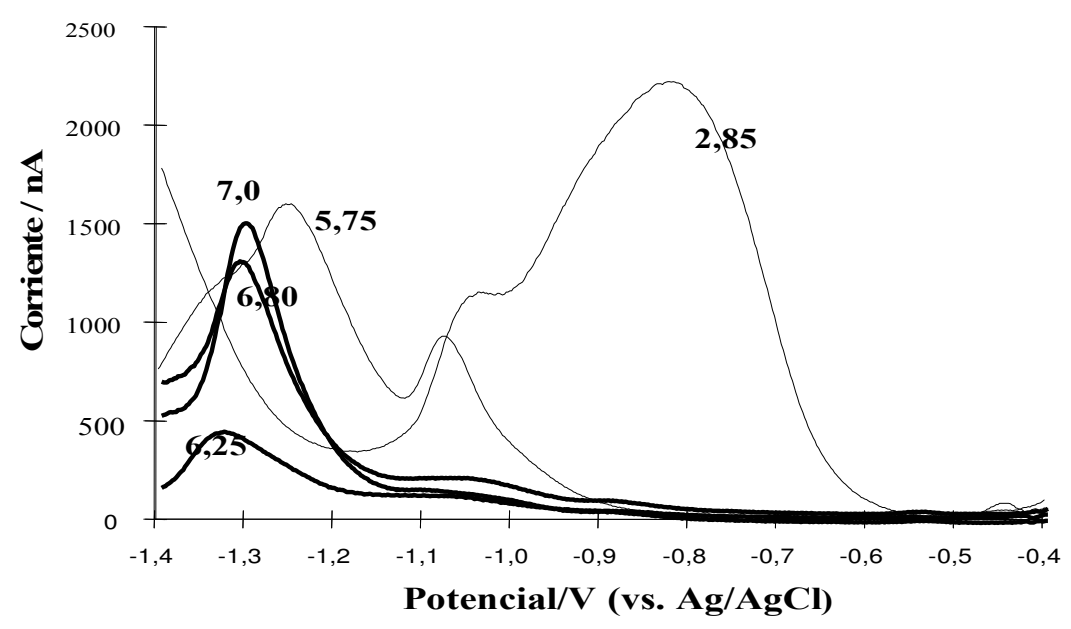

Figura 6. Determinación del $\mathrm{pH}$ adecuado para la formación del complejo aluminio cupferrón. 


\section{Validación del método desarrollado}

El método desarrollado se validó a través de la determinación de sus límites de detección y cuantificación, ámbito lineal, comprobación de la veracidad del método. En la Fig. 9 se determinó la linealidad de las curvas de calibración hasta $60 \mu \mathrm{g} / \mathrm{L}$ de aluminio adicionados en celda; este ámbito de concentración se consideró adecuado para las concentraciones de aluminio en los fluidos empleados en hemodiálisis.

Se determinaron de la Fig. 8 los límites de detección y cuantificación a partir de la desviación estándar de las curvas de calibración [10,11].

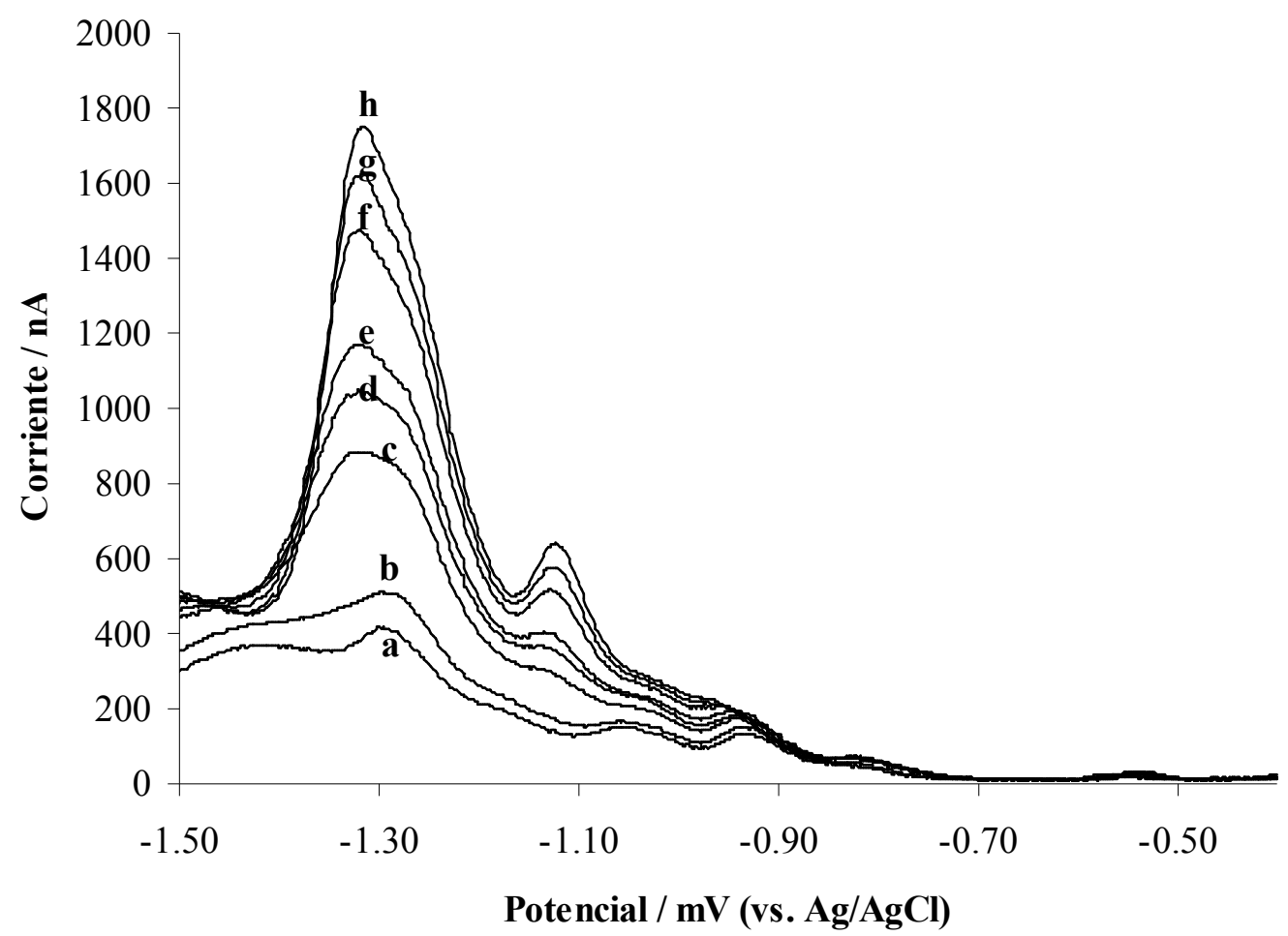

Figura 7. Variación de la corriente al adicionar aluminio en la celda; a. $2 \mu \mathrm{g} / \mathrm{L}$, b. 4 $\mu \mathrm{g} / \mathrm{L}$, c. $8 \mu \mathrm{g} / \mathrm{L}$, d. $10 \mu \mathrm{g} / \mathrm{L}$, e. $12 \mu \mathrm{g} / \mathrm{L}$, f. $16 \mu \mathrm{g} / \mathrm{L}, \mathrm{g} .18 \mu \mathrm{g} / \mathrm{L}, \mathrm{h} .20 \mu \mathrm{g} / \mathrm{L}$.

\section{Determinación de la veracidad del método}

Se mineralizaron con $\mathrm{HNO}_{3}$ disoluciones acuosas por cuadruplicado, a las cuales se le adicionaron 0.4 y $20 \mu \mathrm{g} / \mathrm{L}$ de aluminio, se sometieron a digestión y se analizaron por el método desarrollado, se restó el promedio de los blancos al 
promedio de muestras, la recuperación obtenida para $4 \mu \mathrm{g} / \mathrm{L}$ fue $150 \%$, la recuperación obtenida para $20 \mu \mathrm{g} / \mathrm{L}$ fue $98,2 \%$. Debido al valor alto de las disoluciones de baja concentración, fue necesario utilizar un procedimiento de limpieza de cristalería, reflujando con $\mathrm{HCl} / \mathrm{HNO}_{3} /$ destilados sobre cuarzo en la proporción 3/1. Los parámetros de desempeño del método se muestran en Tabla 3.

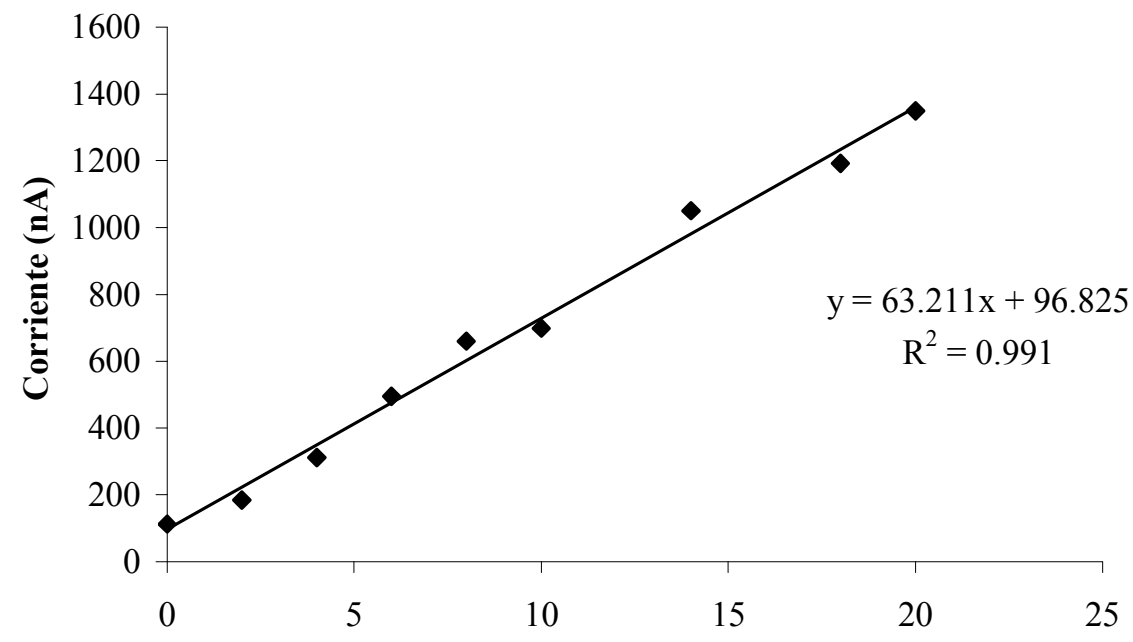

Aluminio adicionado $(\mu \mathrm{g} / \mathrm{L})$

Figura 8. Determinación del límite de detección y cuantificación para el complejo aluminio- cupferrón.

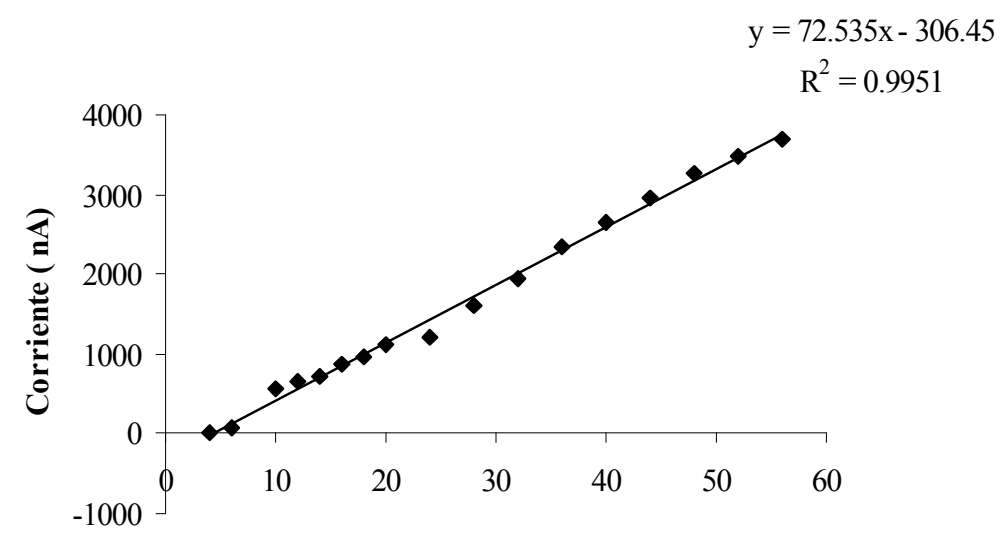

Concentración de aluminio adicionado

Figura 9. Comprobación de la linealidad para el complejo aluminio-cupferrón. 
Tabla 3. Parámetros de desempeño del método voltamperométrico aluminio-cupferrón.

\begin{tabular}{|l|c|}
\hline Límite de detección & $2 \mu \mathrm{g} / \mathrm{L}$ \\
\hline Límite de cuantificación & $7 \mu \mathrm{g} / \mathrm{L}$ \\
\hline Ámbito lineal & $60 \mu \mathrm{g} / \mathrm{L}$ \\
\hline Veracidad $\mathbf{2 0} \boldsymbol{\mu g} / \mathbf{L} \mathbf{n}=\mathbf{7}$ & $98 \%$ \\
\hline Veracidad $4 \boldsymbol{\mu g} / \mathbf{L} \mathbf{n}=\mathbf{7}$ & $150 \%$ \\
\hline Coeficiente de variación en muestras & $17 \%$ \\
\hline
\end{tabular}

\section{Estudio de adicionar $\mathrm{Fe}^{3+}$ como interferencia}

Se construyó una curva de calibración adicionando a un blanco de reactivos diversas concentraciones de aluminio hasta $36 \mu \mathrm{g} / \mathrm{L}$, se le adicionó $\mathrm{Fe}^{3+}$ y se observó el efecto sobre la señal de aluminio; se observó que la gota tendía a saturarse cerca de $60 \mu \mathrm{g} / \mathrm{L}$ de $\mathrm{Fe}^{3+}$, como se muestra en Fig. 10.

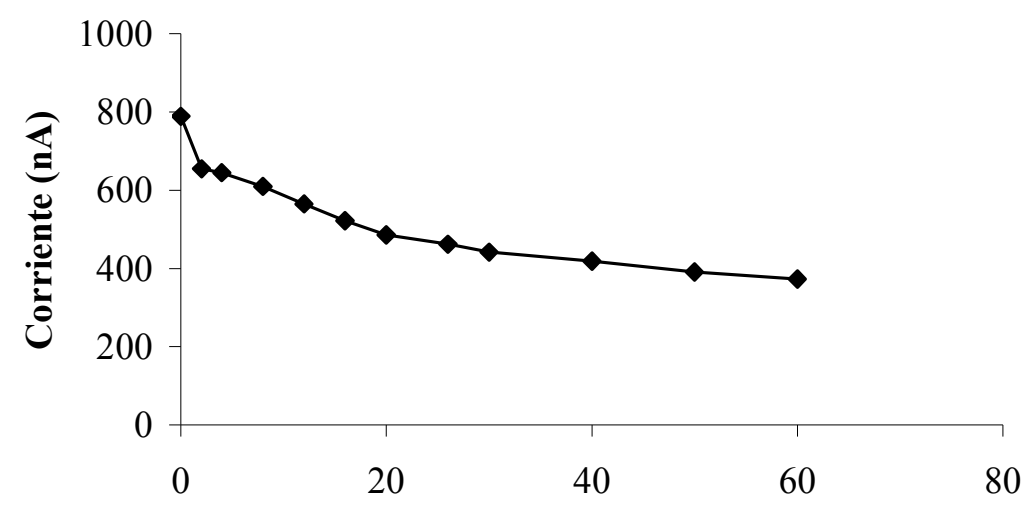

Concentración de hierro (III) adicionado a $36 \mu \mathrm{g} / \mathrm{L}$ de aluminio

Figura 10. Efecto de la interferencia del $\mathrm{Fe}^{3+}$ sobre la señal de $36 \mu \mathrm{g} / \mathrm{L}$ de $\mathrm{Al}^{3+}$ acomplejado con cupferrón.

\section{Estudio de adicionar $\mathrm{Cr}^{3+}$ como interferencia}

Se construyó una curva de calibración adicionando a un blanco de reactivos diversas concentraciones de aluminio hasta $36 \mu \mathrm{g} / \mathrm{L}$, se le adicionó $\mathrm{Cr}^{3+}$ y se observó el efecto sobre la señal; se observó que la gota tendía a saturarse cerca de $5 \mu \mathrm{g} / \mathrm{L}$ de $\mathrm{Cr}^{3+}$. Esto parece indicar que el cromo es una interferencia electroactiva más importante para el complejo aluminio-cupferrón que el hierro, como se ve en la Fig. 11. 


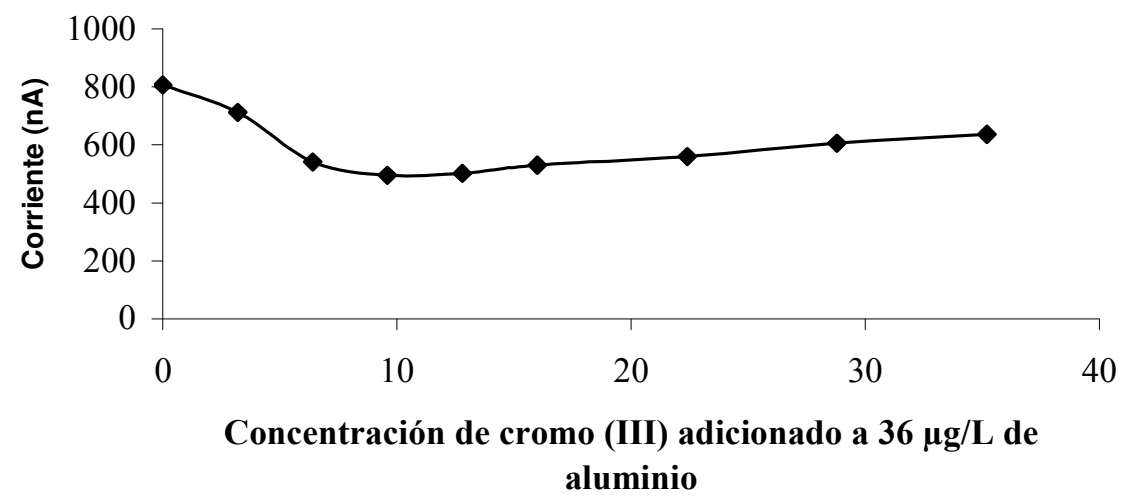

Figura 11. Efecto de la interferencia del $\mathrm{Cr}^{3+}$ sobre la señal de $36 \mu \mathrm{g} / \mathrm{L}$ de $\mathrm{Al}^{3+}$ acomplejado con cupferrón.

\section{Análisis de muestras reales}

El análisis de muestras de fluidos empleados en la hemodiálisis se realizó previo lavado del material de nalgeno con $\mathrm{HNO}_{3}$ al 20\% v/v; se adicionaron $100 \mu \mathrm{L}$ de $\mathrm{HNO}_{3}$ de calidad S.P. al recipiente antes de tomar la muestra. Ésta estaba diluida a la concentración apropiada, para el tratamiento de los pacientes en las maquinas dializadoras. Se empacaron en doble bolsa plástica y se rotularon. Se transportaron rápidamente hasta el laboratorio, se almacenaron en congelación a $-30{ }^{\circ} \mathrm{C}$. Se midieron $4.00 \mathrm{~mL}$ de muestra y se colocaron en material descontaminado: los balones de $25.00 \mathrm{~mL}$ se trataron con $\mathrm{HCl}$ y $\mathrm{HNO}_{3}$ destilados sobre cuarzo, en la proporción $3 / 1$, se calentaron a ebullición y se lavaron con agua Milli-Q. Se mineralizaron las muestras, se aforaron, se midió una masa de muestra y se colocó en la celda polarográfica, se reguló el $\mathrm{pH}$ de la muestra a 7.0 con ayuda de un pHmetro y disoluciones de HOAC, NaOAC, 2 mol/L y NaOH 1 mol/L, según fuera necesario. El control del $\mathrm{pH}$ para las muestras fue muy estricto para formar el complejo. Se adicionó una alícuota de $40 \mu \mathrm{L}$ de cupferrón, se ajustó con agua Milli-Q a una masa final de 10.00 gr y se cuantificó por adición estándar. Los resultados se muestran en la Tabla 4. 
Tabla 4. Resultados de las muestras de fluidos de hemodiálisis analizados.

\begin{tabular}{|l|c|l|c|}
\hline Muestra & Concentración $\boldsymbol{\mu g} / \mathbf{L}$ & Muestra & Concentración $\boldsymbol{\mu g} / \mathbf{L}$ \\
\hline $\mathbf{1 3}$ & & $\mathbf{3}$ & \\
\hline Réplica 1 & D & Réplica 1 & 44.6 \\
\hline Réplica 2 & D & Réplica 2 & 13.4 \\
\hline $\mathbf{5}$ & & $\mathbf{1}$ & 74.6 \\
\hline Réplica 1 & 14.9 & Réplica 1 & \\
\hline Réplica 2 & 10.9 & $\mathbf{2}$ & 72.02 \\
\hline $\mathbf{4}$ & & Réplica 1 & \\
\hline Réplica 1 & 18.9 & $\mathbf{9}$ & 322 \\
\hline $\mathbf{1 2}$ & & Réplica 1 & 392 \\
\hline Réplica 1 & N.D. & Réplica 2 & 34.7 \\
\hline Réplica 2 & N.D. & $\mathbf{8}$ & \\
\hline $\mathbf{1 1}$ & & Réplica 1 & \\
\hline Réplica 1 & 25.6 & $\mathbf{6}$ & 24.0 \\
\hline Réplica 2 & 40.4 & Réplica 1 & \\
\hline & & Réplica 2 & \\
\hline
\end{tabular}

Los límites de detección de $2 \mu \mathrm{g} / \mathrm{L}$ y cuantificación de $7 \mu \mathrm{g} / \mathrm{L}$ resultaron apropiados para los niveles encontrados en los fluidos usados para hemodiálisis, la mayoría de los fluidos analizados superan los $10 \mu \mathrm{g} / \mathrm{L}$ recomendados por AAMI, por lo que sería conveniente el empleo de un tratamiento apropiado al agua utilizada para diluir los concentrados empleados. Las muestras son concordantes al nivel de concentración determinado.

$\mathrm{El} \mathrm{Cr}^{3+}$ se constituye en una interferencia importante porque disminuye en bajas concentraciones la señal de aluminio-cupferrón a su potencial electroactivo.

$\mathrm{El} \mathrm{Fe}^{3+}$ es también una interferencia en el análisis. El ámbito de $\mathrm{pH}$ apropiado para la medición es muy estrecho por lo que en las muestras mineralizadas debe regularse con pHmetro. La cristalería se constituye en una fuente muy importante de contaminación por lo que para tener blancos limpios se deben limpiar los balones empleados para digerir las muestras poniéndolos ebullición con $\mathrm{HNO}_{3}$ y $\mathrm{HCl}$ destilados sobre cuarzo $(1+3)$. La matriz de los fluidos empleados en hemodiálisis es muy compleja por lo que sería provechoso analizar otros cationes presentes a nivel de trazas. 


\section{Agradecimientos}

Se agradece al Dr. Carlos Chavarri Montero y a la Lic. Rosario Vargas y al personal de la unidad de hemodiálisis del Hospital México por su colaboración para el suministro y la toma de las muestras. Se agradece a la Vicerrectoría de Investigación, a la Escuela de Química y al Centro de Electroquímica y Energía Química (CELEQ), de la Universidad de Costa Rica, por el apoyo prestado a este proyecto.

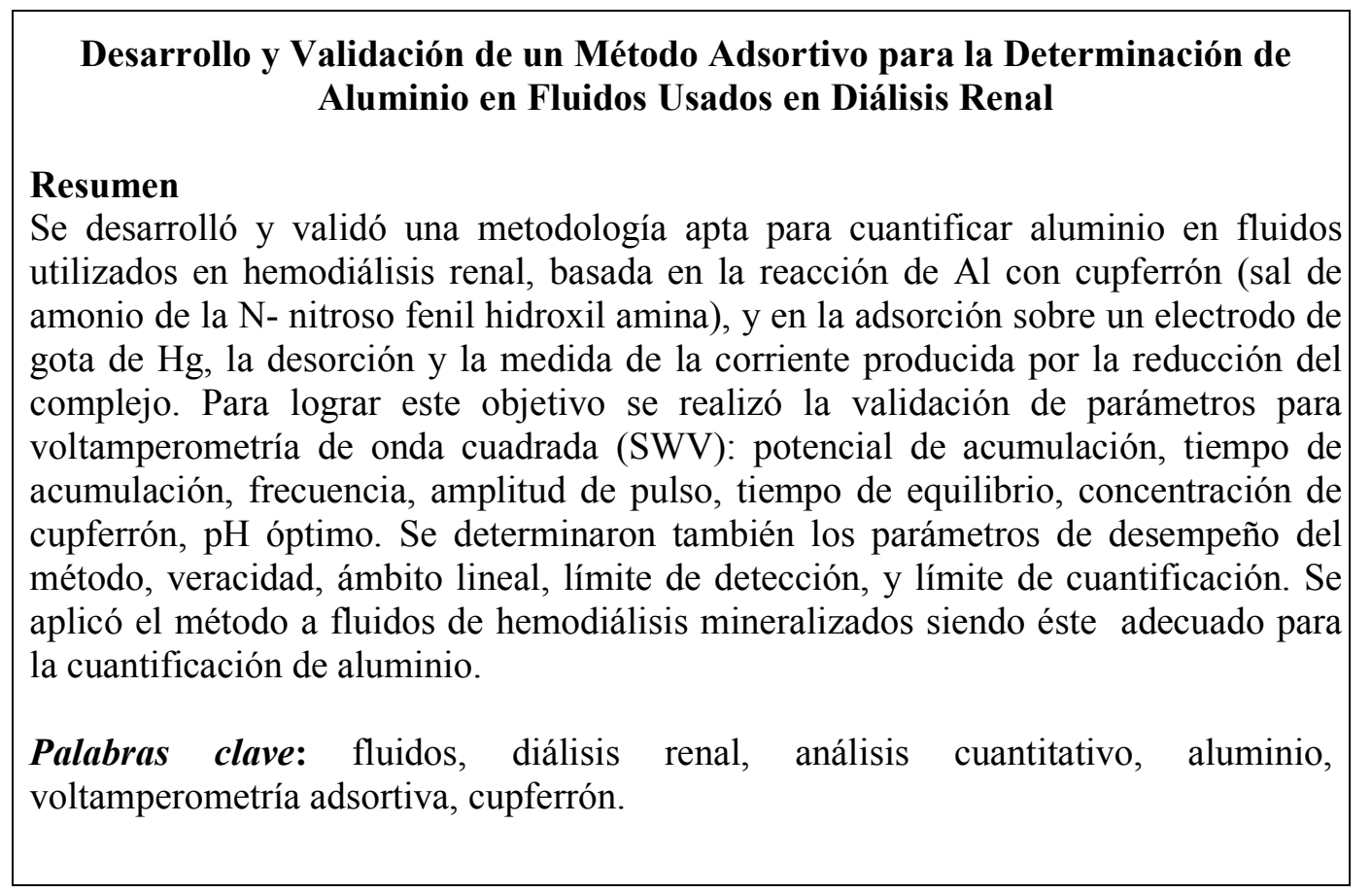

\section{Referencias}

1. R. Vanholder, R. Cornelis, A. Dhondt, N. Lameire, Nephrol. Dial. Transplant. 17 [Suppl. 2] (2002) 2-8.

2. European Renal Association - European Dialysis and Transplant Association, Nephrol. Dial. Transplant. 17 [ Suppl 7] (2002) 45-62.

3. M. Arvanitodou, S. Spaia, P. Tsoubaris, C. Katsinas, N. Askepidis, Dial. Transplant. 29-9 (2000) 519-525.

4. M. Baly', B. Syndel, T. Arynsoy, G. Güz, E. Hasanodlu, Gazi. Med. Journal 6 (1995) 17-2. 
5. Association for Advancement of Medical Instrumentation, AAMI Standards and Recommended Practices, (Vol 3), Arlington, VA American National Standards Institute, 1993.

6. J. Wang, J. Lu, R. Setiadji, Talanta 40-3 (1993) 351-354.

7. M. Silva, B. Freire dos Reis, Quim. Nova 25-6 (2002) 931-934.

8. A. Romero, J. Tahán, A. Moronta, Analytica Chimica Acta 257 (1992) 147.

9. R.C. León, Tesis de Doctorado 2002, Universidad de Extremadura, España.

10. J.N. Miller, J.C. Miller, $4^{\mathrm{a}}$ edición, Estadistica y Quimiometría para Química Analítica, Prentice Hall, 2000, Capítulo 5.

11. J.K. Taylor, Quality Assurance of Chemical Measurements, Lewis Publisher Inc., $2^{\text {nd }}$ printing, USA, 1987. 